\title{
Ultrathin four-reflection imager
}

\author{
Eric J. Tremblay, ${ }^{1, \star}$ Ronald A. Stack, ${ }^{2}$ Rick L. Morrison, ${ }^{2}$ \\ Jason H. Karp, ${ }^{1}$ and Joseph E. Ford ${ }^{1}$ \\ ${ }^{1}$ Department of Electrical and Computer Engineering, University of California, San Diego, \\ La Jolla, California 92093, USA \\ ${ }^{2}$ Distant Focus Corporation, Champaign, Illinois 61820-7460, USA \\ *Corresponding author: etremblay@ucsd.edu
}

Received 22 September 2008; revised 3 December 2008; accepted 4 December 2008;

posted 5 December 2008 (Doc. ID 101823); published 7 January 2009

\begin{abstract}
We present the design and experimental demonstration of an ultrathin four-reflection imager. The $F / 1.15$ prototype imager achieves a focal length of $18.6 \mathrm{~mm}$ in a track length of just $5.5 \mathrm{~mm}$, providing a $17^{\circ}$ field of view over 1.92 megapixels of a color image sensor with $3 \mu \mathrm{m}$ pixels. We also present the design and experimental results of pupil-phase encoding and postprocessing, which were applied to extend the depth of field and compensate a small amount of axial chromatic aberration present in the fourreflection imager prototype. (C) 2009 Optical Society of America

OCIS codes: $\quad 080.3620,110.0110,100.2000,220.1920$.
\end{abstract}

\section{Introduction}

The need for compact visible-light imagers has led to extensive use of short focal length, small aperture lenses that meet the strict space and weight constraints required for many commercial and military applications. While these miniature refractive lenses often perform well in good lighting and within a small range of optical magnification, resolution and light collection are limited by the focal length and physical track length of the lens. One method of significantly increasing focal length and magnification without a corresponding increase in track length consists of reflecting the optical path multiple times with concentric reflectors, thus constraining the optical propagation to occur within a thin optical element. This concept is based on an extension of traditional reflective telescope design [1,2] with additional reflectors to minimize track length and an enlarged diameter to maintain light collection [3].

This thin multiple concentric reflector approach leads to an optical lens design with large surface powers, a large obscuration ratio, and tight fabrication tolerances. These challenges can be met by

0003-6935/09/020343-12\$15.00/0

(C) 2009 Optical Society of America
(1) the use of multiple aspheric reflectors optimized to correct the aberrations caused by the geometry, (2) an enlarged diameter to allow multiple concentric reflections, improve light collection, and offset the diffraction effects caused by the annular aperture, and (3) self-alignment of multiple surfaces through use of single-point diamond turning (SPDT) to fabricate concentric annular aspheric reflectors with minimal rechucking. An eight-reflection imager was previously demonstrated experimentally in [3] by diamond turning a single side of a calcium fluoride $\left(\mathrm{CaF}_{2}\right)$ lens blank and coating the substrate with patterned silver reflectors. The eight-reflection imager of [3] achieved an effective focal length (EFL) of $35 \mathrm{~mm}$ in a physical track of just $5 \mathrm{~mm}$, providing a well-corrected image over a field of view (FOV) of $6.67^{\circ}$.

The steep ray angles present in a highly obscured multiple-reflector design create a shallow depth of field (DOF) [4]. Several hybrid optical-digital approaches have been investigated for increasing the DOF of imaging systems [5-11]. These approaches optimize the amplitude and $\bar{d} / \mathrm{or}$ phase of the imaging system's pupil function to create optical systems that are invariant with respect to defocus over a given range. Digital image restoration techniques are then used to restore image contrast lost by the pupil 
modification. Wavefront coding, the hybrid opticaldigital approach of Dowski and Cathey [5], utilized a cubic-phase pupil function for extended DOF. This approach is attractive since it is compatible with incoherent illumination and its phase-only modification of the pupil function maintains light throughput better than pupil function apodization approaches. In recent years, more general phase-only masks and several design approaches utilizing different metrics in the frequency and spatial domain have been introduced for defocus invariance [9-11]. Previously we examined the application of an annular cosineform pupil-phase function optimized in the frequency domain for use with an eight-reflection lens design to relax the image-sensor alignment tolerances [12]. Although experimental fabrication errors caused significant deviation from the design, we measured a modest improvement in focal plane alignment tolerance and depth of focus.

In this paper we present the design and experimental demonstration of an ultrathin four-reflection imager prototype with a novel and sensitive method of refocus. Compared to the previously demonstrated eight-reflection imager of [3], this four-reflection imager demonstrates a less extreme focal length extension; however, it offers a smaller diameter and volume, smaller obscuration, larger FOV, and larger DOF. These characteristics make it suitable for a wider range of applications. In this paper, Section 2 describes the design and simulation of our four-reflection imager. Section 3 describes the fabrication, integration, and experimental demonstration of the four-reflection imager. Section 4 describes the application of pupil-phase encoding ( $\overline{\mathrm{PPE}})$ to the full aperture four-reflection design and compares the performance to the baseline four-reflection imager design. Finally, Section $\underline{5}$ contains our conclusions.

\section{Four-Reflection Imager Design}

Here we describe a four-reflection imager design intended for use as an ultrathin visible-light camera. The design achieves a FOV of $17^{\circ}$ over 1.92 megapixels of a color image sensor with $3 \mu \mathrm{m}$ pixels in just $5.5 \mathrm{~mm}$ of total track.

\section{A. General Considerations and Design Space}

Figure 1 depicts the general design concept and geometry for a thin multiple-reflection lens and compares a thin paraxial lens of focal length $F$ to an obscured multiple-reflection version using a single thin (paraxial) powered mirror at the first reflection. With this simplified thin reflector geometry of an arbitrary even number of reflections (e.g., 2, 4, $6)$, we can examine and relate the important geometric parameters: FOV, aperture, focal length, diameter, thickness, and number of reflections. This approach is similar to one described in [13], however, the analysis given here describes results given by a powered reflector rather than a refractive lens.

The focal length of the multiple-reflection lens shown in Fig. 1(b) will be $F=N T / n_{s}$, where $F$ is

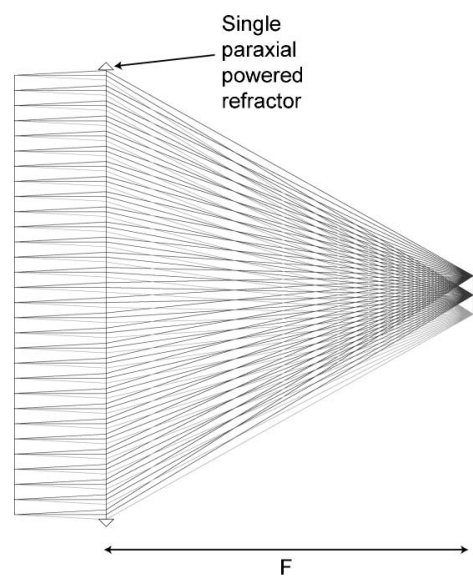

(a)

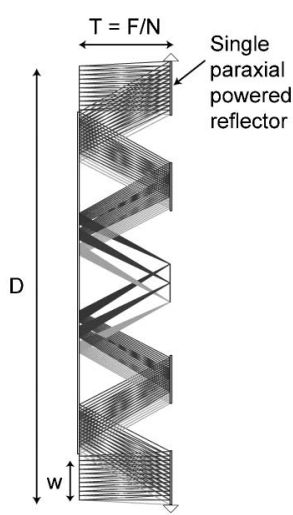

(b)
Fig. 1. Design concept and geometry: (a) thin (paraxial) lens of focal length $F$ and (b) four-reflection obscured version using a single thin (paraxial) reflector of focal length $F$.

the focal length, $N$ is the number of reflections, $T$ is the thickness of the lens, and $n_{s}$ is the refractive index of the image-side medium. For small FOV, we find that, to avoid vignetting losses, the width of the annular aperture is limited by the outermost oblique ray position at the second reflector. This condition allows us to express the size of the aperture as a function of the other geometric parameters as

$$
w=\frac{D}{2 N}-\tan (\mathrm{FOV} / 2) \cdot F \cdot \frac{(2-1 / N)}{N},
$$

where $w$ is the width of the annular aperture, and $D$ is the diameter. For FOV larger than

$$
\mathrm{FOV}=2 \tan ^{-1}\left(\frac{D N}{2 F\left(N^{2}-1\right)}\right),
$$

and for four or more reflections, we find that the width of the aperture is no longer limited at the second reflector, but at the second to last reflector, assuming the image plane is designed to be in contact with the back of the lens. For these large values of FOV, the width of the annulus is given by

$$
w=\frac{D}{2 N}-\tan (\mathrm{FOV} / 2) \cdot F \cdot(N-1+1 / N) .
$$

A value of $w=0$ in Eq. (3) determines the maximum FOV possible where the aperture size has gone to zero. Figure 2 shows a plot of $w$ versus FOV using Eqs. (1) and (3), where a sharp drop in aperture size can be seen once the FOV of Eq. (2) has been met. With $N=2$, the common two-reflector scheme, the aperture will be reduced to zero at the FOV of Eq. (2) (the max FOV), and Eq. (1) alone is sufficient to describe the relationship between parameters.

These relationships do not include diffraction effects or aberrations but are useful in providing general geometric relationships to define potentially 


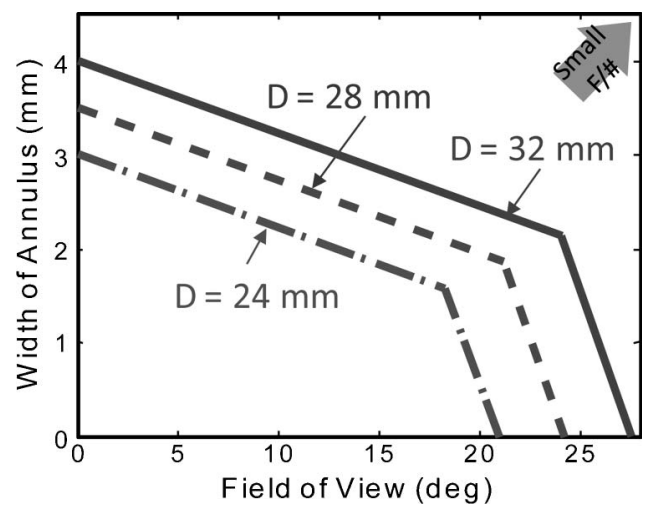

Fig. 2. Example curves of annular aperture width $(w)$ versus FOV for various diameters using paraxial geometric calculations $\left(N=4, F=20 \mathrm{~mm}\right.$, and $n_{s}=1$ in this example). As aperture size in increased, FOV decreases. As diameter is increased while maintaining fixed thickness, number of reflections, and focal length, both aperture and FOV increase.

useful design areas. As in all reflective systems, FOV is limited compared to a comparable refractive lens. Equations (1)-(3) indicate that as the number or reflections $(N)$, focal length $(F)$, or thickness $(T)$ are increased, maximum FOV decreases. The maximum achievable FOV is also increased if an internal dielectric substrate $\left(n_{s}>1\right)$ is used, since the increased index of refraction reduces the focal length (with respect to a system in air), image height, and ray angles within the lens in the same way that focusing a lens into a higher refractive-index medium reduces these properties. In addition, as FOV is increased, the annular aperture size, $w$ must decrease to prevent overlap of the annular reflectors. Finally, as the diameter, $D$, is increased, both the FOV and $w$ increase. This last point is important since it states that we need an enlarged diameter to fit a number of reflections into a thin design with reasonable FOV and aperture. With an enlarged diameter and several reflections, we can expect very large surface power, $\Phi$, and surface "work," $\Phi y$, where $y$ is the ray height off the optical axis on the first reflector. This large surface work will provide significant aberrations that must be corrected for. To do this, each subsequent reflection of a multiple-reflector lens can be used for aberration correction and/or to extend the focal length of the system. Our present designs have required these surfaces to be aspheric due to required surface powers and the severe constraints on thickness, FOV, and EFL. To illustrate this point, the root mean square geometric spot size of the fourreflection lens described in this paper was reduced by a factor of more than 300x using aspheric surfaces compared to the same constrained design using only spherical surfaces. Aspheric surfaces create additional difficulties with tolerances but can be fabricated with little additional effort or cost using SPDT.

Diffraction effects from the highly obscured annular aperture are important since we expect obscuration ratios (defined as the diameter of the obscuration divided by the diameter of the outer diameter) in excess of 0.7. Large obscuration ratios significantly reduce midspatial frequency values of the modulation transfer function (MTF) [14] and are not usually acceptable in reflective telescopes due to their effect on image contrast. In our case we can accept the form of the highly obscured MTF since our diameter is scaled up enough to correct for the otherwise low contrast of the midspatial frequencies. We show in Section 2.B that the Nyquist frequency of

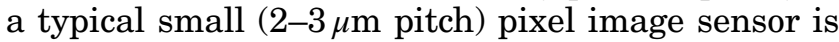
much smaller than the cutoff frequency of the reflective lens's diffraction-limited MTF. For these spatial frequencies of interest, the diffraction-limited MTF will be sufficient for good contrast in our design.

\section{B. Four-Reflection Imager Design: Specific}

Our objective in the design of an ultrathin visiblelight imager was to create a reflective lens design capable of a $17^{\circ} \mathrm{FOV}$, illuminating $1600 \times 12003 \mu \mathrm{m}$ pixels of a color image sensor in a physical track length close to $5 \mathrm{~mm}$. To meet these specifications, a four-reflection lens design in $\mathrm{CaF}_{2}$ was chosen and optimized in ZEMAX EE, a commercially available ray-tracing program. Our four-reflection lens design, shown in Fig. 3(a), is a thin annular reflective lens utilizing four concentric aspheric reflectors to focus incoherent light to an image plane in near alignment with the back side of the lens. The solid geometry (solid dielectric as opposed to air) was chosen to increase the achievable FOV, which is limited by the reflective geometry as described in Subsection 2.A. $\mathrm{CaF}_{2}$ was

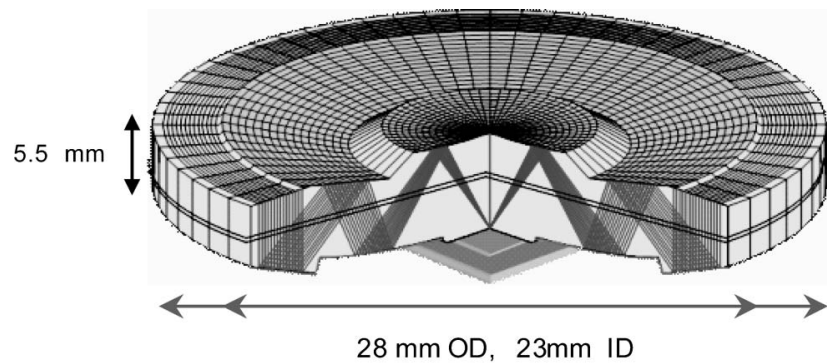

(a)

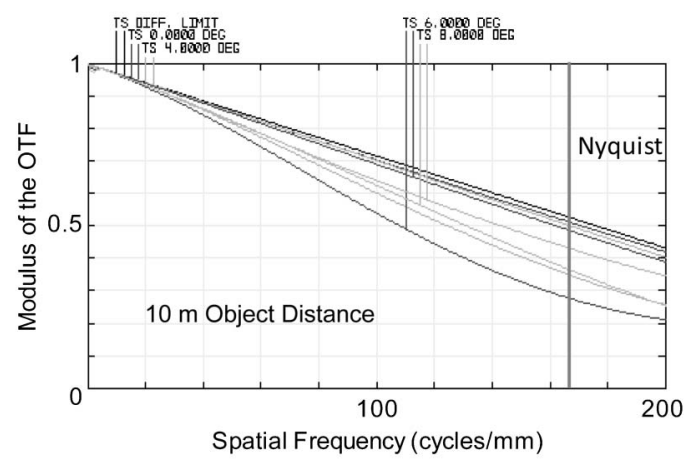

(b)

Fig. 3. (a) Layout drawing (3/4 section) of the four-reflection imager and (b) monochromatic MTF at the nominal design object distance of $10 \mathrm{~m}$. OD, object distance; ID, image distance; OTF, optical transfer function. 
chosen as the substrate material for relatively low dispersion but primarily due to compatibility with SPDT (with $\mathrm{CaF}_{2}$, an optical quality finish is possible without postpolishing). In contrast to the eightreflection lens in [3], the front and back sides of the four-reflection lens are fabricated separately as two plano-aspheric lens elements that are subsequently aligned and joined with index-matching gel between the elements. Focusing is done by varying the nominal $300 \mu \mathrm{m}$ part spacing between these two elements as very large changes in refocus are available with a gap-spacing adjustment of the order of micrometers. Fabrication of the two lens elements separately also avoids a critical alignment in rechucking a single element during fabrication in favor of adjustable alignment in assembly. This arrangement does, however, require the mechanical package to support the focus adjustment while maintaining the critical alignment between the two lens elements.

The four-reflection lens design shown in Fig. 3(a) has an EFL of $18.6 \mathrm{~mm}$ and a numerical aperture (NA) of 0.72 . The effective $F / \#$ (defined as the focal length divided by the equivalent diameter of an unobscured aperture of the same aperture area) is 1.15. The design is $28 \mathrm{~mm}$ in diameter and $5.5 \mathrm{~mm}$ thick with an obscuration ratio of 0.81 . Its $17^{\circ}$ FOV fills a $1600 \times 1200$ pixel color image sensor with $3 \mu \mathrm{m}$ pixels. At the extremes of the field, near the corners of the image sensor, relative illumination is $87 \%$, and pincushion distortion is limited to $7 \%$.

The incoherent modulation transfer function (MTF) is shown in Fig. 3(b). The design performs well with incoherent monochromatic light across the full FOV up to the Nyquist frequency, 167 line pairs per millimeter $(\mathrm{lp} / \mathrm{mm})$ of the image sensor. Across the full visible spectrum (486 to $656 \mathrm{~nm}$ ), up to $10 \mu \mathrm{m}$ of lateral color aberration (at edge of field) is present from dispersion as light enters the $\mathrm{CaF}_{2}$ lens material. In addition, larger axial color aberration than expected was found experimentally due to dispersion in the commercially available Cargille 0608 indexmatching gel. This dispersion has been accounted for in the current simulations and results in a chromatic focal shift of $7 \mu \mathrm{m}$.

Chromatic aberration is not intrinsic to concentric multireflection lens designs in general, since an airspaced catoptric lens with first-surface reflectors would have no chromatic dependences. Even introducing a flat cover glass on an air-spaced design does not introduce significant chromatic aberration. However, the first refraction at the interface of a dielectric-filled lens introduces lateral color aberration that cannot be easily compensated. If packaging constraints or FOV make a dielectric-filled catadioptric lens desirable, it may be possible to omit the index-matching gel in favor of a small adjustable air-gap for focus. In this arrangement, the addition of planar low-power surface-relief diffractive elements fabricated with SPDT can be used to correct the chromatic aberrations incurred from the airdielectric refractions. To help correct the chromatic focal shift present in our current four-reflection imager design, we show in Section 4 simulated and experimental results for the application of PPE to help correct the axial color aberration described earlier by increasing the imaging system's defocus tolerance [15].

\section{Image-Sensor Selection}

The steep incidence angles present at the image plane in this four-reflection lens design can create a problem with detection efficiency with conventional complementary metal-oxide semiconductor (CMOS) image sensors, especially those with small pixels. This issue, often referred to as pixel vignetting, occurs due to shadowing from the metal interconnect layers that surround each pixel [16]. Microlenses are usually employed to help focus light onto the active sensor area. However, microlenses do not function as intended with large NA designs [17], including our reflective lens as well as conventional low $F / \#$ refractive lenses. In our prototype fourreflection imager, the microlenses are therefore covered with a thin layer of index-matching gel, filling the void between the image sensor and fourreflection lens. To reduce loss caused by pixel vignetting, we have chosen a 1.93 megapixel $(1600 \times 1200)$ color CMOS image sensor with 3 micropixels made by Forza/Sunplus. This image sensor, made by the IBM copper-CMOS process, has a relatively small interconnect stack height of $2 \mu \mathrm{m}$. With this image sensor, we found a $70 \%$ improvement in marginal ray efficiency as compared to a more conventional CMOS image sensor with $3.6 \mu \mathrm{m}$ aluminum interconnects. Further improvement is possible with the use of back-side illuminated (BSI) image sensors, which completely eliminate the obstruction caused by metal interconnects on the image sensor's surface. BSI image sensors are currently being developed to improve performance of high pixel count CMOS image sensors with low $F / \#$ refractive optics [18].

\section{Packaging}

A mechanical package and support structure was designed to meet the requirements of the fourreflection imager. The package was designed to hold the front and back lens elements in precise alignment in order to maintain optical performance while allowing the gap spacing between them to be adjusted for refocus as shown in the cutaway view in Fig. 4. The material used for the package was 303 stainless steel, which is similar in thermal expansion characteristics to the $\mathrm{CaF}_{2}$ lens material. This reduces unwanted strain on the lens elements that could degrade optical performance. The front lens element is designed to be glued into the main enclosure body while the back lens element is designed to be glued into a back lens carrier. The back lens carrier also serves as a mounting point for the image-sensor circuit board and provides a moderate amount of tip/tilt adjustment for the circuit board as well. A cover snaps onto the back of main enclosure 


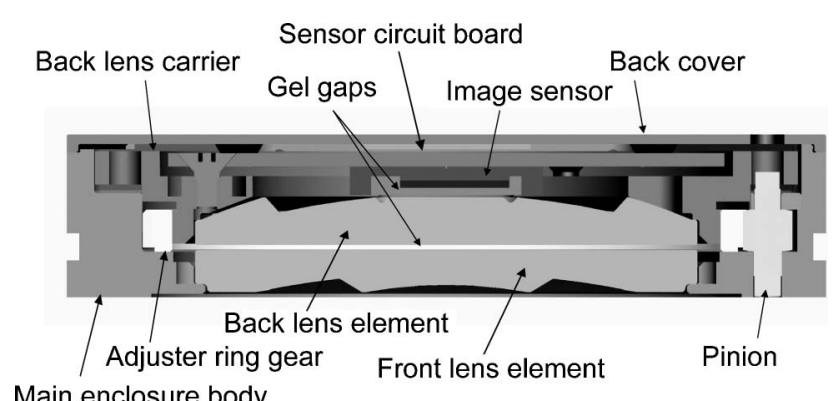

Main enclosure body

Fig. 4. Cross-section drawing of the mechanical lens package.

body to protect internals. The back lens carrier slides into a precision-machined bore in the main enclosure body to maintain the $5 \mu \mathrm{m}$ alignment needed between the two elements. To set the position of the back lens carrier, and thus the gap spacing between the two lens elements, a 168-tooth (120 pitch) gear adjustment ring is threaded onto precision threads cut into the back lens carrier. The adjustment ring rides on a machined surface located in the main enclosure body. A small 12-tooth pinion allows for rotation of the adjustment ring using a small screwdriver, where one rotation of the pinion screw corresponds to an adjustment in the gap spacing of $28 \mu \mathrm{m}$. Four springs clamp the back lens carrier into the main enclosure body and provide the clamping force needed to expel the index-matching gel when adjusting lens elements closer together.

\section{E. Refocus}

The adjustable gap between the two lens elements of the four-reflection imager allows focus to be adjusted for different object ranges via small adjustments around the nominal $300 \mu \mathrm{m}$ lens element gap spacing. MTF curves for the imager focused at $4 \mathrm{~m}$ and $1 \mathrm{~km}$ are shown in Figs. 5(a) and 5(b), respectively. Some degradation of the tangential component of the most extreme field angles can be seen in Fig. 5(a) for close object distance; however, results are generally excellent across a very large range of refocus. The total adjustment for this refocus is $14 \mu \mathrm{m}$. The small travel of this focus adjustment method may be especially suitable for use with a precision actuator such as a piezoelectric transducer. For our experimental purposes and to simplify the design, we have chosen a mechanical adjustment using a pinion-gear reduction assembly to adjust the spacing between the two lens elements as described in Subsection 2.D. The main drawback of our chosen method is the tight $5 \mu \mathrm{m}\left(0.0002^{\prime \prime}\right)$ bore tolerance between the main enclosure body and the back lens carrier that needs to be maintained. This turned out to be very difficult for the machine shop to consistently achieve, even with matched sets of the parts. In order to ensure the back lens carrier slides smoothly in its machined bore during a refocus, a certain amount of clearance was required, and in practice, this clearance was larger than $5 \mu \mathrm{m}$. This ultimately led to problems with misalignment when a refocus was performed
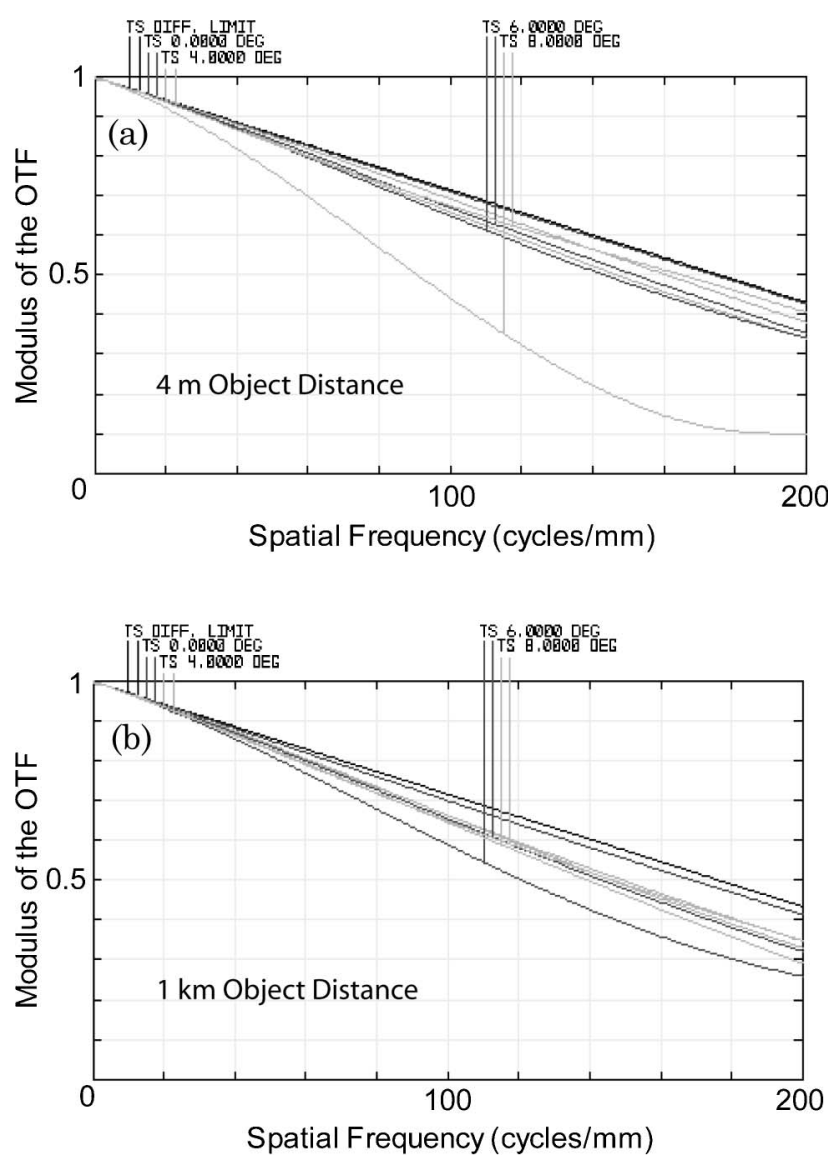

Fig. 5. Simulated refocus performance of the four-reflection lens design. Monochromatic MTF curves at object distances of (a) $4 \mathrm{~m}$ and (b) $1 \mathrm{~km}$. A $14 \mu \mathrm{m}$ gap adjustment is required to focus from position (a) to position (b). OTF, optical transfer function.

due to rear lens carrier shifting in the bore. Future designs will most likely use a flexure style focusing mechanism actuated with a piezoelectric transducer.

\section{F. Fabrication and Assembly Tolerances}

The four-reflection lens was intended to be fabricated as two plano-aspheric lens elements to allow for focus adjustment by varying the gap spacing between the front and back lens elements. While this geometry relieves the critical alignment involved with rechucking a single double-sided lens during SPDT as well as the critical part thickness tolerance present in the previously reported eight-reflection lens of [3], it presents additional difficulties with the assembly of the two lens elements once they have been fabricated. The tolerances of the shifts and tilts of the aspheric surfaces are similar to those of the eightreflection lens with minimum values of $\pm 10 \mu \mathrm{m}$ for surface shift (along the optic axis) and $\pm 0.01^{\circ}$ surface tilt. The overall lens element thickness tolerance is $\pm 50 \mu \mathrm{m}$, and the tolerable departure from the aspheric surface equation is $\pm 0.25 \lambda$ (at $546 \mathrm{~nm}$ ) within each annular reflector zone. Once fabricated, the two lens elements must be carefully aligned in the mechanical package for centration and tilt. The assembled centration and tilt tolerances are $5 \mu \mathrm{m}$ 
(radial) and $\pm 0.02^{\circ}$, respectively. These tolerances were calculated using ZEMAX's sensitivity analysis to maintain an MTF greater than $10 \%$ at 167 cycles $/ \mathrm{mm}$ across the full field at the nominal object distance of $10 \mathrm{~m}$.

\section{Four-Reflection Prototype}

Here we describe the fabrication and assembly of a four-reflection imager for experimental demonstration. Once the lens elements were fabricated using SPDT, we aligned and glued the elements into a mechanical package designed to allow for mechanical focus adjustment. Experimental results of the assembled imager are presented and discussed.

\section{A. Fabrication and Assembly}

ISP Optics [19] diamond turned the front and back plano-aspheric $\mathrm{CaF}_{2}$ lens elements and coated them with patterned dielectric reflectors as shown in Fig. 6(a). The specified fabrication tolerances for these elements were achieved on the first pass. Once fabricated, we aligned and glued the two reflective lens elements with UV epoxy into the mechanical package shown in Fig. 6(b). This process was carried out on a measuring microscope to carefully align the elements for centricity and tilt. The front lens element was first centered and epoxied into the main enclosure body. The completed front lens assembly was then mounted to the microscope with blocking glue, and the rear lens element was placed directly on top of front lens assembly. The direct contact resulted in zero gap spacing between the lens element's planar sides to ensure minimal tilt. The back lens carrier was then placed into position with the adjustment ring set appropriately. We then aligned the centers of the front and back lens elements to within the centration tolerance of $5 \mu \mathrm{m}$ with the measuring microscope and affixed the rear lens element to the back lens carrier with epoxy. The assemblies were then disassembled from the alignment station and reassembled with index-matching gel filling the nominal $300 \mu \mathrm{m}$ gap spacing between the two lens elements.

Finally, we aligned and mounted the image-sensor circuit board onto the back lens carrier assembly. The spacing between the transmissive aperture of the

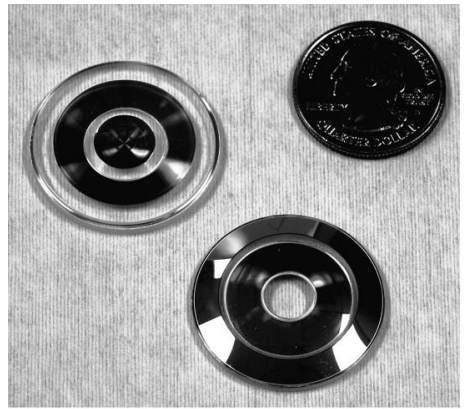

(a)

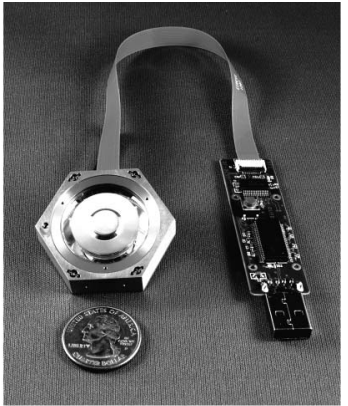

(b)
Fig. 6. Four-reflection imager assembly: (a) diamond turned and coated optical parts and (b) image of the assembled four-reflection imager with USB interface PCB. rear lens element and the image-sensor surface was set to $0.6 \mathrm{~mm}$. This spacing was constrained primarily by the necessary chip package clearance. Indexmatching gel was used to fill the gap between the rear lens element and the image sensor to remove the effect of the microlenses. The imager assembly connects to a computer through a ribbon cable connected to the USB interface as shown in Fig. 6(b). Image-sensor settings, image capture, and processing are controlled by MDOSim, a custom interactive camera programming environment written in $\mathrm{Py}$ thon and developed by Distant Focus Corporation.

\section{B. Measured Performance}

A full-field $1600 \times 1200$ image at an object distance of $3.9 \mathrm{~m}$ is shown in Fig. 7(a). The exposure time for the image is $35 \mathrm{~ms}$ under typical fluorescent laboratory illumination. Examining the United States Air Force (USAF) resolution chart, which can be seen more closely in Fig. 7(b), we find the limit of resolution to be group $(-\overline{1,4)}$ or $0.707 \mathrm{lp} / \mathrm{mm}$ in object space at this range. This corresponds to a measured angular resolution of $0.363 \mathrm{mrad}$.

Figure 7(c) shows the measured contrast transfer function $(\overline{\mathrm{CTF}})$ (lens + sensor) for the four-reflection imager at $3.9 \mathrm{~m}$. CTF measurements were made by first carefully white balancing the image of a resolution chart and then measuring the various bar-chart contrast values referenced to the white and black levels at low spatial frequency. The image sensor's raw Bayer image data were used to avoid any additional loss of contrast or resolution from the default color interpolation process. The CTF of the four-reflection imager shows a resolution cutoff of approximately $150 \mathrm{lp} / \mathrm{mm}$ in image space. For comparison, the CTF of a conventional refractive Sanyo SVCL-CS550VM $F / 1.4$ zoom lens mounted to the same image sensor is shown as well in Fig. 7(c). The zoom lens focal length has been set to $1 \overline{9 \mathrm{~mm}}$ to match the focal length of the four-reflection imager lens. The Sanyo lens resolution is image-sensor limited with a measured resolution of $\sim 175 \mathrm{lp} / \mathrm{mm}$. This measured cutoff frequency is larger than the Nyquist frequency of the image sensor due to additional bandwidth around the fundamental frequency of the three-bar image [20].

The measured CTF results of the four-reflection imager lens are close to those predicted by optical simulation when the dispersion of the indexmatching gel and measured decentration of the final assembled imager are included. The measured $5 \mu \mathrm{m}$ decentration between the two assembled lens elements corresponds to the limit or tolerance, where detrimental effects on image quality at best focus start to become significant.

With the same image-sensor settings and averaged pixel gains as the four-reflection imager, the conventional comparison lens and image sensor required an exposure of $17 \mathrm{~ms}$ to image the resolution targets with similar levels. The larger exposure required by the four-reflection imager can be attributed to losses 
of the dielectric reflectors and residual pixel vignetting at the image sensor. Transmission through the four-reflection imager lens was measured with a collimated green laser and large area silicon detector to be $38 \%$, which is significantly lower than the designed transmission of $>90 \%$. Assuming a second fabrication run would achieve the design performance in mirror reflectivity, the sensitivity of the four-reflection imager will be identical to that achieved with the long conventional refractive lens.

Figure 7(d) shows the measured DOF of the fourreflection imager lens and the conventional comparison lens. With both lenses focused at $3.9 \mathrm{~m}$, images of the USAF resolution charts were taken at various distances to gauge the fall off in resolution as a function of distance. As measured, the DOF of the fourreflection imager lens is roughly $3 \times$ smaller than the conventional lens. This difference is smaller than the approximate DOF difference predicted by $1 / \mathrm{NA}^{2}$ due to the large obscuration and, in part, to the nature of the measurement. The large obscuration causes an annular blur, which, for small values of defocus, does not affect resolution as significantly as an unobscured aperture of the same NA. However, for large values of defocus, the annular blur causes a rapid and sudden falloff in resolution and perceived image quality due to the annular bokeh [21].

\section{Stray Light}

Reflective imaging systems are extremely susceptible to stray light since rays may encounter reflective surfaces nonsequentially and appear as noise at the image sensor. Reflective telescopes typically use large hoods and internal field stops as primary and secondary baffles to limit the angular extent of the light reaching the image sensor $[22,23]$. These structures are often long compared to the physical size of the lens and are not desirable when creating an ultrathin imager. A complete analysis of the origins of stray light in the four-reflection imager is necessary to develop appropriate baffling techniques.

We performed systematic stray light calculations using ZEMAX nonsequential analysis to identify critical surfaces that can be seen directly by the image sensor. Tracing rays in reverse from the image sensor to the input aperture is helpful to recognize specific paths where light can directly reach the image sensor [24]. Multiple reflections occurring within thin reflective lens designs lead to extreme ray angles that may enter the system and strike incorrect facets or skip

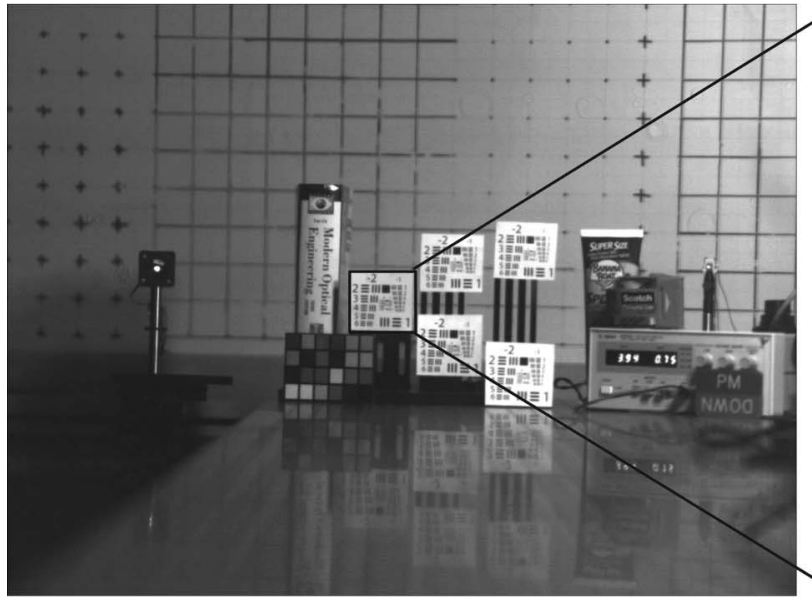

(a)

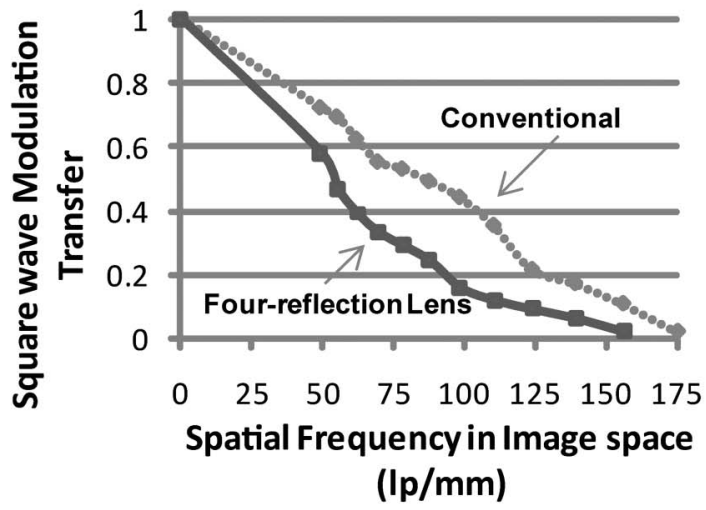

(c)

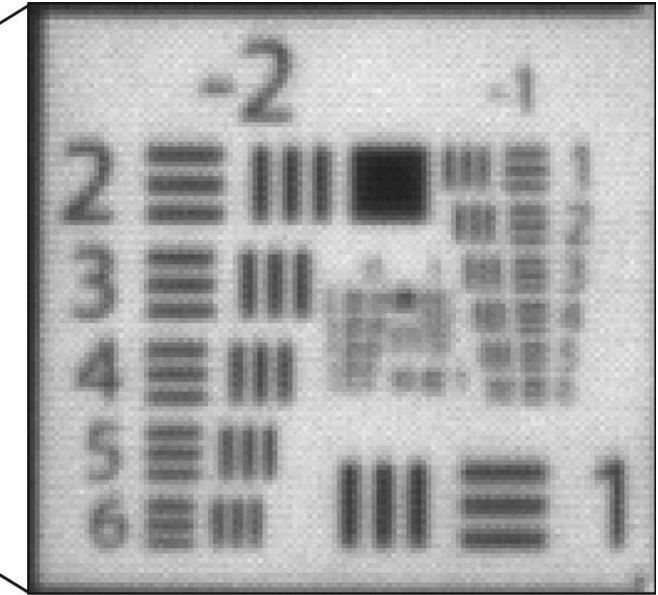

(b)

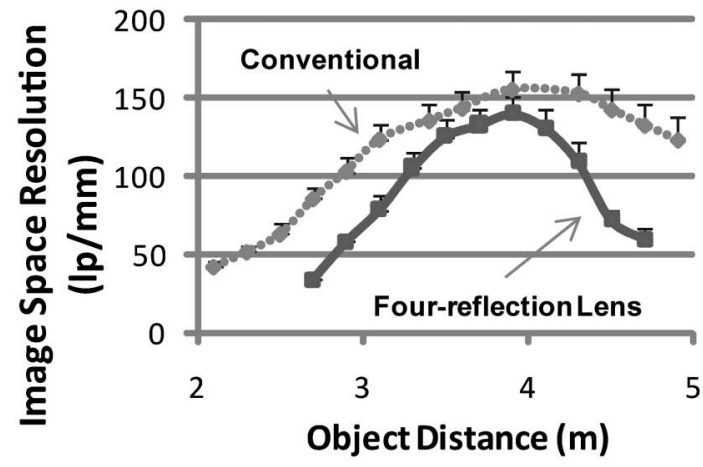

(d)

Fig. 7. Performance at $3.9 \mathrm{~m}$ : (a) full image, (b) enlarged and cropped 1951 USAF resolution chart, (c) measured CTF (lens + sensor) of the four-reflection imager compared to a conventional $F / 1.4$ lens of the same focal length and sensor, and (d) image space resolution versus object distance for the four-reflection imager and conventional $F / 1.4$ comparison imager. 
surfaces altogether. Skew rays tend to migrate around the exit pupil and do not contribute to significant levels of stray light.

We confirmed the stray light simulations experimentally by rotating the four-reflection imager with respect to a fixed light source. The four-reflection imager has a designed $8.5^{\circ}$ half-angle FOV. Significant amounts of light at incidence angles beyond $12^{\circ}$ off axis reach the image sensor. These errant paths appear as bright bands at specific locations within the field and can lead to complete image loss. An example of bright oblique stray light imaged by the image sensor is shown in Fig. 8(a). Additionally, an annular gap exists between the two surfaces cut into the front lens element and provides a direct ray path to the detector without encountering any of the appropriate surfaces. This effect is shown in Fig. 8(b). The reflector of the front lens element must be covered to prevent light leaking through this front surface onto the image sensor. Figure 8(c) shows simulated and measured normalized intensity versus incidence angle for the four-reflection imager, indicating the most problematic stray light paths to the image sensor.

A simple hood placed on the front of the fourreflection imager would have to extend $190 \mathrm{~mm}$ to limit incident rays to the design FOV. This excessive length can be shortened by dividing the full $28 \mathrm{~mm}$ aperture into small subapertures, each limiting the FOV. We found a thin, commercial honeycomb baffle made by Tenebraex to be a suitable angle-selective

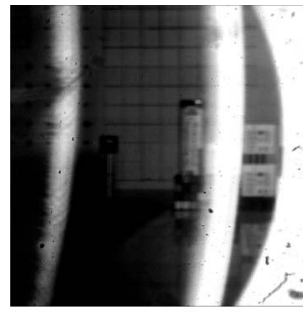

(a)

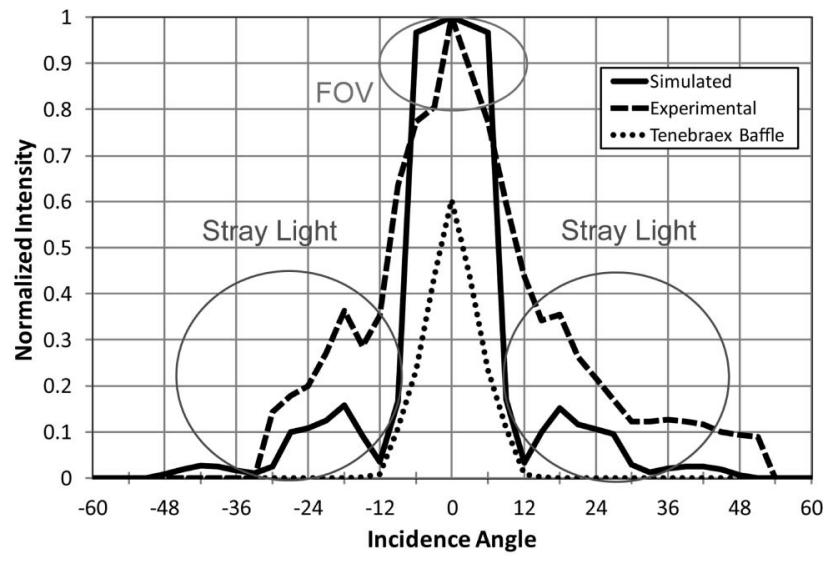

(c)

Fig. 8. Stray light in the four-reflection imager: (a) large angle oblique rays skip reflectors and arrive at the sensor as stray light, (b) axial light may enter through gaps in the front reflectors if a central block is not used, and (c) simulated and measured normalized intensity versus incidence angle. baffle for our four-reflection imager. The baffle has a $\pm 12^{\circ}$ angular cutoff and reduces the overall baffle length to $6.3 \mathrm{~mm}$. Placing the honeycomb in front of the aperture causes a $40 \%$ drop in on-axis transmission since vanes now block portions of the entrance pupil. Off-axis illumination is linearly attenuated down to the cutoff angle. A central obscuration is placed within the honeycomb to block the direct stray light path through the annular gap. By controlling stray light paths to the image sensor with this baffle, we have been able to use the camera in brightly lit situations, including direct sunlight.

Other aspects of image degradation associated with intense illumination are seen within the fourreflection imager. Chromatic flare from the dielectric mirror coating can be seen at some stray light angles, especially for rays entering the annular gap. Scatter from the honeycomb vanes can also reduce the contrast of the image. Very bright spots formed on the image sensor produce surface reflections that reenter the folded track. These rays may reflect from the last aspheric reflector back to the image sensor, leading to a narcissuslike effect of thermal imagers, where the camera sees itself.

The honeycomb baffle works well for stray light suppression; however, despite its reduced thickness, it is still thicker than the four-reflection imager lens. More extreme versions of the subaperture baffling concept are being explored using capillary arrays and even high-resolution holographic film to expose the desired baffling structure. The resulting devices can be only micrometers thick, although care will be necessary to minimize scatter and loss.

\section{Application of Pupil-Phase Encoding and Postprocessing}

Here we describe the design and experimental implementation of PPE and postprocessing for the fourreflection imager. We investigate this technique for extending the DOF of the four-reflection imager while simultaneously correcting axial chromatic aberration.

\section{A. Design of Phase-Modulated Surface}

We applied PPE to the four-reflection imager by modifying the nominally flat annular aperture on the front side of the four-reflection lens. The large obscuration ratio presents challenges for the application of the phase-modulated surface since pupilphase modulation is limited along the radial coordinate. It has been previously shown [12] that the cosine form of phase modulation is well suited to such optical designs with large obscuration ratio. This is due to significant modulation possible in the cosinusoidal angular component along with a smaller radial component. The cosine form is also well suited to fabrication with SPDT with a fast-tool servo to shape the periodic structure of the rotationally asymmetric surface. The general shape of the cosine form is described as 


$$
\operatorname{Sag}(r, \theta)=\sum_{i=1}^{m} a_{i} r^{b_{i}} \cos \left(w_{i} \theta+\varphi_{i}\right),
$$

where $r, \theta$, and $\operatorname{Sag}(r, \theta)$ specify the surface position and shape in cylindrical coordinates. The weight on each term is given by $a_{i}$, the powers of the radial terms are given by $b_{i}$, the radian frequency is given by $w_{i}$, and the phase terms are given by $\varphi_{i}$. In our four-reflection lens design, we optimized the specific surface figure given by

$$
\begin{aligned}
& \operatorname{Sag}(r, \theta)=\sum_{i=1}^{8} a_{i}(r-0.81)^{i} \cos (3 \theta) \\
& \quad \text { for }\{0.81 \leq r \leq 1.0\},
\end{aligned}
$$

where the range of $r$ values represent a scaled radius from the limiting radius of obscuration through the outer aperture radius. This surface profile is applied to the nominally planar front annular aperture of the four-reflection lens.

The goal of our optimization was to extend the depth of focus of our four-reflection imager to $\pm 10 \mu \mathrm{m}$, thus increasing the depth of focus of the four-reflection imager by a factor of approximately $3 \times$ and creating a defocus tolerance large enough to compensate for the axial color aberration present in our design. We carried out the optimization using a combination of commercial ray-tracing software, ZEMAX, and commercial numerical computing software, MATLAB. An intermediate software link provided us with the flexibility to incorporate our own image-sensor modeling, postprocessing, and optimization routines in MATLAB, with calls to ZEMAX to perform the necessary ray traces through our four-reflection lens design. We defined a custom merit function to optimize the optical system such that a single restoration filter could be applied to increase the depth of focus. The goals of the merit function were (1) to maximize the average MTF values across the fields, focal plane positions, and wavelengths of the design up to the Nyquist frequency of the intended image sensor, (2) to reduce the variation between MTFs across the fields, focal plane positions, and wavelengths, and (3) to maintain a minimum threshold MTF for all fields, focal plane positions, and wavelengths up to the Nyquist frequency of the intended image sensor. Our merit function to be minimized for optimum system performance can be described as

$$
\text { merit value }=\text { offset }-\alpha_{1} A+\alpha_{2} B+\alpha_{3} C,
$$

where $\alpha_{1}, \alpha_{2}, \alpha_{3}$ are the weights on the mean, standard deviation, and minimum threshold terms, respectively, and offset is a user-specified term to maintain a positive merit function value. The mean term $A$ is defined as

$$
A=\operatorname{mean}_{c, f, \lambda}\left(\operatorname{mean}_{u x, u y}\left(\operatorname{MTF}_{c, f, \lambda}\left(u_{x}, u_{y}\right)\right)\right),
$$

where $c$ is the configuration (focal plane position), $f$ is the field position, $\lambda$ is the wavelength, and $u_{x}$ and $u_{y}$ are the spatial-frequency coordinates. Because of symmetry, only the first quadrant of the $\mathrm{MTF}_{c, f, \lambda}$ $\left(u_{x}, u_{y}\right)$ function is needed in the optimization calculations. In Eq. (7), mean indicates the arithmetic mean. Since a large mean value is desirable, this term is assigned negative value in the merit function, Eq. (6). The standard deviation term, $B$, in Eq. (ㅁ) is described as

$$
B=\operatorname{mean}_{u x, u y}\left(\operatorname{stdev}_{c, f, \lambda}\left(\operatorname{MTF}_{c, f, \lambda}\left(u_{x}, u_{y}\right)\right)\right) .
$$

Here MTF values are compared across the configurations, fields, and wavelengths to enforce similarity between optimized MTF values. The arithmetic mean is used to average the standard deviation values over the spatial-frequency coordinates to give a single value to the merit value equation. Finally, the threshold term, $C$, in Eq. (6) is described as

$$
C=\operatorname{mean}_{c, f, \lambda}\left(\beta_{c, f, \lambda}\right),
$$

where

$\beta_{c, f, \lambda}=\left\{\begin{array}{ll}\left(\frac{\delta_{\text {thresh }}-\mathrm{MTF}_{\min ;: f, \lambda}, \lambda}{\left(\delta_{\text {thresh }} / 10\right)}\right)^{n} & \text { if }\left(\frac{\delta_{\text {thresh }}-\mathrm{MTF}_{\min : c, f, \lambda}}{\left(\delta_{\text {thresh }} / 10\right)}\right)>0 . \\ 0 & \text { otherwise }\end{array}\right.$.

In Eq. (10), $\delta_{\text {thresh }}$ is the chosen minimum MTF value threshold, $n$ is the exponent power on the threshold term, and $\mathrm{MTF}_{\min : c . f . \lambda}$ is the calculated minimum MTF value within the first quadrant for each configuration, field position, and wavelength up to the Nyquist frequency of the image sensor. Depending on the value of the minimum MTF value below threshold, Eq. (10) is designed to give positive values between zero and ten raised to the $n$th power. This term in the merit value therefore enforces a minimum MTF across the configurations, field positions, and wavelengths for image restoration and penalizes zero crossings where image information is lost and phase inversion occurs.

We carried out optimization of the PPE surface using the merit value described by Eqs. (6)-(10), utilizing several different optimization routines such as the Nelder-Mead simplex (direct search) method, gradient/finite difference, and simulated annealing to investigate solutions offered by the different methods [25-27]. The weights of the terms in Eq. (6) were adjusted to investigate their influence on the results of optimization. In general, the standard deviation term, $B$, and the minimum threshold term, $C$, received much stronger weighting than the mean term, $A$. The threshold term in the merit value equation was chosen to maintain a modulation of greater than $10 \%$ up to the Nyquist frequency of our sensor ( 167 cycles $/ \mathrm{mm}$ ) across the operating range. Typically, a modulation of $10 \%$ is sufficient 
for effective image restoration with postprocessing. We found the simplex method in general to be the most useful and reliable in terms of convergence and speed; however, significant interaction was required, and a large number of optimization starting points were tried to steer the optimization toward a usable solution. Modeled results of our final solution are displayed in Fig. 9, where the axial point spread function (PSF) over $\pm \overline{1} 0 \mu \mathrm{m}$ defocus is shown for both the unmodified and PPE designs. A simple inverse filter is used to generate the processed PSFs shown in the bottom row of Fig. 9 [28]. For display purposes, the intensity scale on the unmodified PSFs (top row) and the PPE PSFs (middle and bottom rows) differ by a factor of $\sim 4 \times$. This value represents the trade-off between signal-to-noise ratio (SNR) and depth of focus with this technique. The trade-off is minimized as much as possible in the optimization by maximizing the average MTF across configurations, fields, and wavelengths; however, the significant variation between field positions in the baseline four-reflection lens design required a relatively strong phase modulation, lower average MTF, and more significant trade-off of SNR to achieve our desired spatial invariance over the image volume (depth of focus and DOF) $[29,28]$.

\section{B. Experimental Results}

To demonstrate the optimized PPE surface, we had the front element of the four-reflection lens refabricated with the optimized PPE surface profile diamond turned onto the previously flat annular aperture. The modified front element was then assembled with the standard rear element as described

Axial unmodified PSFs

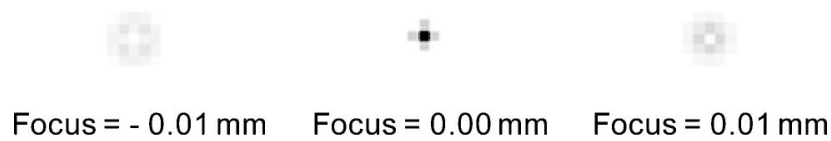

Axial Phase-Encoded PSFs w/o Processing
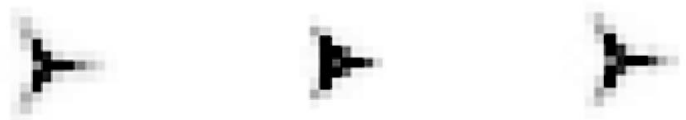

Focus $=-0.01 \mathrm{~mm} \quad$ Focus $=0.00 \mathrm{~mm} \quad$ Focus $=0.01 \mathrm{~mm}$

Axial Phase-Encoded Processed PSFs

Focus $=-0.01 \mathrm{~mm} \quad$ Focus $=0.00 \mathrm{~mm} \quad$ Focus $=0.01 \mathrm{~mm}$ Fig. 9. Simulated PSFs at best focus and $\pm 10 \mu \mathrm{m}$ defocus $( \pm 3.5$ waves defocus at $550 \mathrm{~nm}$ ). Unmodified PSFs (top row), PPE PSFs before filtering (middle row), and inverse-filtered PPE PSFs (bottom row). in Section 3 to make a PPE four-reflection imager. To test the addition of the PPE surface, we set up a comparison test with the PPE four-reflection imager and the unmodified four-reflection imager at the same object distance. Figure 10 shows axial PSFs measured with both the unmodified four-reflection imager and the PPE four-reflection imager. We measured the PSF by imaging a $25 \mu \mathrm{m}$ pinhole illuminated by a bright white-light LED positioned $3.9 \mathrm{~m}$ away from the two imagers. Best focus was found by adjusting the pinion screw that varies the separation of the front and back optical elements until the most compact PSF was found. We measured PSFs at best focus and $\pm 0.3 \mathrm{~m}$ to characterize the PSF variations through focus for the two imagers. The measured object depth of $0.6 \mathrm{~m}$ corresponds to a depth of approximately $20 \mu \mathrm{m}$ (or \pm 3.5 waves of defocus at $550 \mathrm{~nm}$ ) in image space. Examining the PSFs for the unmodified imager, we found that, although the imager forms a small bright PSF at best focus, the out-of-focus measured PSFs display an asymmetry associated with the measured decentration of $5 \mu \mathrm{m}$ between the lens elements. In this case, we find the PSF at $3.6 \mathrm{~m}$ to be better than the simulated results shown in Fig. 9 . The asymmetry in the PSF leads to significant power concentration on a small PSF, as the object distance is reduced from best focus. However, this behavior is not symmetric through focus increasing the object distance from best focus caused the PSF to spread more quickly on the far side of best focus. The PSFs

\section{Axial Unmodified PSFs}

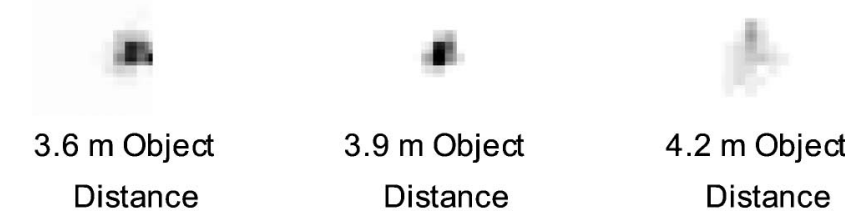

Axial Phase-Encoded PSFs w/o Processing

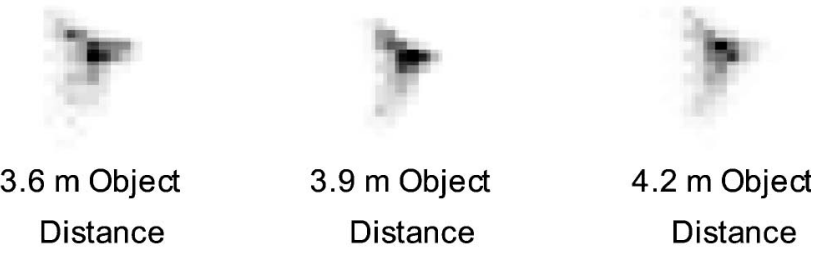

Axial Phase-Encoded Processed PSFs

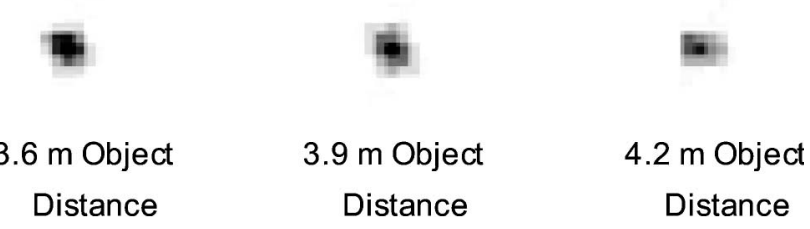

Fig. 10. Measured PSFs at $3.9 \mathrm{~m}$ (best focus) and $\pm 0.3 \mathrm{~m}(\sim \pm 3.5$ waves defocus at $550 \mathrm{~nm}$ ). Unmodified PSFs (top row), PPE PSFs before filtering (middle row), and inverse-filtered PPE PSFs (bottom row). 
for the PPE four-reflection imager also show some asymmetry from a measured decentration of $11 \mu \mathrm{m}$ (assembly error) between the lens elements. This decentration is large enough to cause significant degradation in image quality in the PPE four-reflection imager. The decentration tolerance for the PPE fourreflection lens is approximately $8 \mu \mathrm{m}$. Using matched pixel gains and sensor settings, the exposure times used for the PSF measurements of Fig. 10 are 0.78 and $3.3 \mathrm{~ms}$ for the unmodified and PPE fourreflection imagers, respectively. The bottom row in Fig. 10 shows filtered results of the PPE fourreflection imager where a common Wiener filter has been applied to restore contrast [28]. This Wiener filter is based on the measured axial PSF at $3.9 \mathrm{~m}$, with its threshold and SNR balance parameters tweaked to subjectively provide the best-looking images based on a human observer's viewpoint.

Figure 11 shows comparison images of a USAF 1951 resolution chart acquired with the unmodified and PPE four-reflection imagers at best focus $(3.9 \mathrm{~m})$. The color images of the PPE imager were white balanced and converted to luminance-bandwidthchrominance image data before the restoration filter was applied to the $Y$ channel only. At best focus, the resolution limit is $0.707 \mathrm{lp} / \mathrm{mm}$ in the horizontal and vertical directions for the unmodified imager and is 0.63 and $0.561 \mathrm{lp} / \mathrm{mm}$ in the vertical and horizontal directions, respectively, for the PPE imager. The Wiener filter was able to restore much of the resolution of the PPE system; however, the significant misalignment of the lens elements resulted in some resolution loss in the horizontal direction. The noise evident in Fig. 11(b) is introduced by the restoration filter due to the significant noise gain of the filter.

Figure 12 shows another comparison of the unmodified and PPE four-reflection imagers with the object distance reduced by $30 \mathrm{~cm}$ to $3.6 \mathrm{~m}$. At this position, the resolution of the unmodified imager is found to have fallen to $0.561 \mathrm{lp} / \mathrm{mm}$ in the vertical direction and $0.500 \mathrm{lp} / \mathrm{mm}$ in the horizontal direction. The PPE imager, however, shows little perceivable resolution loss over this distance, still resolving $0.63 \mathrm{lp} / \mathrm{mm}$ in the vertical direction and $0.561 \mathrm{lp} / \mathrm{mm}$ in the horizontal direction.

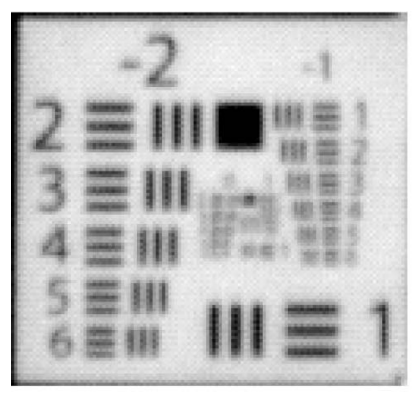

(a)

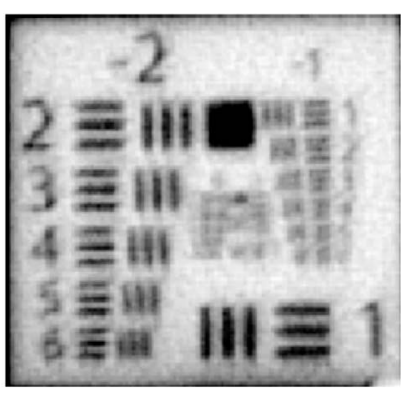

(b)
Fig. 11. Experimental comparison images at $3.9 \mathrm{~m}$ (best focus): (a) unmodified imager and (b) processed PPE imager.

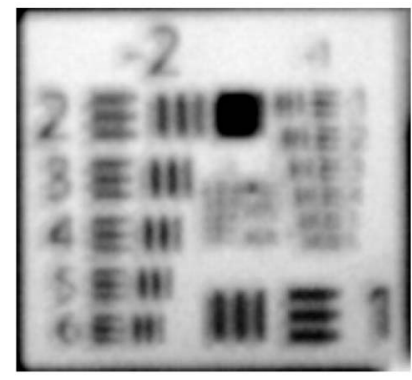

(a)

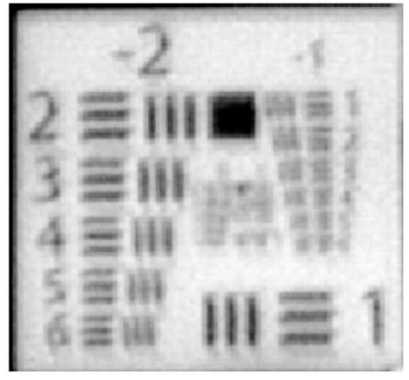

(b)
Fig. 12. Experimental comparison images at $3.6 \mathrm{~m}$ ( $\sim 3.5$ waves defocus at $550 \mathrm{~nm}$ ): (a) unmodified imager and (b) processed PPE imager.

Increasing the object distance by $30 \mathrm{~cm}$ to $4.2 \mathrm{~m}$ created a more pronounced difference between the two four-reflection imagers. At $4.2 \mathrm{~m}$, the unmodified imager's quality is degraded, resolving $0.315 \mathrm{lp} / \mathrm{mm}$ in the vertical direction and $0.353 \mathrm{lp} / \mathrm{mm}$ in the horizontal direction. In contrast, the PPE imager shows a smaller amount of degradation resolving $0.561 \mathrm{lp} / \mathrm{mm}$ in the vertical direction and $0.445 \mathrm{lp} / \mathrm{mm}$ in the horizontal direction.

With the variations in assembly alignment of the two imager systems, it is difficult to directly quantify the improvement gained with PPE and postprocessing in this case. However, even with a measurably larger decentration in the PPE four-reflection imager $(11 \mu \mathrm{m})$ compared to the unmodified four-reflection imager $(5 \mu \mathrm{m})$, we find some improvement in the DOF. Additional effort is required to improve alignment accuracy to more clearly examine the benefit of PPE and postprocessing.

\section{Conclusion and Discussion}

We presented the design and successful experimental demonstration of an ultrathin four-reflection imager that achieves a focal length of $18.6 \mathrm{~mm}$ and effective $F / \#$ of 1.15 in a track length of just $5.5 \mathrm{~mm}$. This reflective lens provides a $17^{\circ} \mathrm{FOV}$ over $1600 \times 12003 \mu \mathrm{m}$ pixels of a color CMOS sensor which is integrated with USB connection to computer. The four-reflection lens and CMOS sensor are mated together within a stainless steel package that provides a sensitive focus adjustment with small changes of the order of a few micrometers to the nominal $300 \mu \mathrm{m}$ spacing between the front and back lens elements. Despite a small chromatic focal shift caused by commercially available index-matching gel and imperfect alignment in the optical assembly, the self-contained and packaged four-reflection imager performed close to the optical design performance under laboratory conditions. Direct comparison using an identical image sensor mated to a large conventional lens of the same focal length showed that resolution and subjective image quality are comparable between the two lenses despite the dramatically reduced track length of the four-reflection imager lens. 
We also investigated the application of PPE and postprocessing for extending the DOF and alleviating the axial chromatic aberration of the four-reflection imager. Although we expected a pronounced improvement in defocus invariance from design and simulation, we found the experimental PSFs for the PPE imager to be significantly different from simulation due to misalignment of the optical assembly. Nevertheless, we found a discernable improvement in DOF in the processed PPE images compared to those of the unmodified four-reflection imager.

Looking forward, such multiple-reflection lenses may be suitable for inexpensive mass production using a glass molding process provided in part that the design include additional registration surfaces to aid in the alignment during assembly. As shown, centration is of particular importance and must be strictly controlled to within a few micrometers for good optical performance.

The authors acknowledge M. Stenner and M. Neifeld (University of Arizona) and R. Athale and D. Healy (Darpa) for many useful technical discussions. We acknowledge ISP Optics for fabrication services. This research was supported by the Defense Advanced Research Projects Agency (DARPA) via the MONTAGE program, grant HR0011-04-I-0045 and by the Natural Sciences and Engineering Research Council of Canada (NSERC) through a graduate student scholarship.

\section{References}

1. W. J. Smith, Modern Lens Design (McGraw-Hill, 2005), Chap. 18.

2. D. Korsch, Reflective Optics (Academic, 1991).

3. E. J. Tremblay, R. A. Stack, R. L. Morrison, and J. E. Ford, "Ultra-thin cameras using annular folded optics," Appl. Opt. 46, 463-471 (2007).

4. J. Hall, " $F$-number, numerical aperture, and depth of focus," in Encyclopedia of Optical Engineering (Marcel Dekker, 2003), pp. 556-559.

5. E. R. Dowski, Jr. and W. T. Cathey, "Extended depth of field through wavefront coding," Appl. Opt. 34, 1859-1866 (1995).

6. W. T. Cathey and E. Dowski, "A new paradigm for imaging systems," Appl. Opt. 41, 6080-6092 (2002).

7. K. Kubala, E. Dowski, J. Kobus, and R. Brown, "Aberration and error invariant space telescope systems," Proc. SPIE 5524, 54-65 (2004).

8. K. Kubala, E. Dowski, and W. T. Cathey, "Reducing complexity in computational imaging systems," Opt. Express 11, 2102-2108 (2003).

9. W. Chi and N. George, "Electronic imaging using a logarithmic asphere," Opt. Lett. 26, 875-877 (2001).
10. S. Prasad, T. C. Torgersen, V. P. Pauca, R. J. Plemmons, and J. van der Gracht, "Engineering the pupil phase to improve image quality," Proc. SPIE 5108, 1-12 (2003).

11. S. Prasad, V. P. Pauca, R. J. Plemmons, T. C. Torgersen, and J. van der Gracht, "Pupil-phase optimization for extended focus, aberration corrected imaging systems," Proc. SPIE 5559, 335-345 (2004).

12. E. J. Tremblay, J. Rutkowski, I. Tamayo, P. E. X. Silveira, R. A. Stack, R. L. Morrison, M. A. Neifeld, Y. Fainman, and J. E. Ford, "Relaxing the alignment and fabrication tolerances of thin annular folded imaging systems using wavefront coding," Appl. Opt. 46, 6751-6758 (2007).

13. M. W. Haney, "Performance scaling in flat imagers," Appl. Opt. 45, 2901-2910 (2006).

14. V. N. Mahajan, "Imaging with obscured pupils," Opt. Lett. 1, 128-129 (1977).

15. H. B. Wach, E. R. Dowski, Jr., and W. T. Cathey, "Control of chromatic focal shift through wavefront coding," Appl. Opt. 37, 5359-5367 (1998).

16. P. B. Catrysse and B. A. Wandell, "Optical efficiency of image sensor pixels," J. Opt. Soc. Am. A 19, 1610-1620 (2002).

17. G. Agranov, V. Berezin, and R. H. Tsai, "Crosstalk and microlens study in a color CMOS image sensor," IEEE Trans. Electron Devices 50, 4-11 (2003).

18. M. Dirjish, "BSI technology flips digital imaging upside down," http://electronicdesign.com/Articles/Index.cfm?AD= $1 \&$ ArticleID $=19160$.

19. http://www.ispoptics.com/.

20. D. H. Kelly, "Spatial frequency, bandwidth, and resolution," Appl. Opt. 4, 435-435 (1965).

21. T. Ang, Dictionary of Photography and Digital Imaging: the Essential Reference for the Modern Photographer (Watson-Guptill, 2002).

22. R. Prescott, "Cassegrainian baffle design," Appl. Opt. 7, 479-481 (1968).

23. C. Leinert and D. Klüppelberg, "Stray light suppression in optical space experiments," Appl. Opt. 13, 556-564 (1974).

24. G. Peterson, "Stray light calculation methods with optical ray trace software," Proc. SPIE 3780, 132-137 (1999).

25. J. C. Lagarias, J. A. Reeds, M. H. Wright, and P. E. Wright, "Convergence properties of the Nelder-Mead simplex method in low dimensions," SIAM J. Optim. 9, 112-147 (1998).

26. T. F. Coleman and Y. Li, "An interior, trust region approach for nonlinear minimization subject to bounds," SIAM J. Optim. 6, 418-445 (1996).

27. S. Kirkpatrick, C. D. Gelatt, and M. P. Vecchi, "Optimization by simulated annealing," Science 220, 671-680 (1983).

28. H. C. Andrews and B. R. Hunt, Digital Image Restoration (Prentice-Hall, 1977), Chap. 8, pp. 147-152.

29. B. R. Frieden, "Image enhancement and restoration," in Topics in Applied Physics, Vol. 6 of Picture Processing and Digital Filtering, T. S. Huang, ed. (Springer-Verlag, 1979), pp. 177-248. 